\title{
A substellar mass function for Alpha Persei ${ }^{\star}$
}

\author{
D. Barrado y Navascués ${ }^{1}$, J. Bouvier ${ }^{2}$, J. R. Stauffer ${ }^{3}$, N. Lodieu ${ }^{4}$, and M. J. McCaughrean ${ }^{4}$ \\ 1 Laboratorio de Astrofísica Espacial y Física Fundamental, INTA, PO Box 50727, 28080 Madrid, Spain \\ 2 Laboratoire d'Astrophysique, Observatoire de Grenoble, Université Joseph Fourier, BP 53, 38041 Grenoble Cedex 9, France \\ e-mail: jerome.bouvier@obs.ujf-grenoble.fr \\ 3 IPAC, California Institute of Technology, Pasadena, CA 91125, USA \\ e-mail: stauffer@ipac.caltech.edu \\ 4 Astrophysikalisches Institut Potsdam, An der Sternwarte 16, 14482 Potsdam, Germany \\ e-mail: nlodieu@aip.de,mjm@aip.de
}

Received 18 March 2002 / Accepted 28 August 2002

\begin{abstract}
We present a deep, wide-field optical survey of the young stellar cluster Alpha Per, in which we have discovered a large population of candidate brown dwarfs. Subsequent infrared photometric follow-up shows that the majority of them are probable or possible members of the cluster, reaching to a minimum mass of $0.035 M_{\odot}$. We have used this list of members to derive the luminosity and mass functions of the substellar population of the cluster $(\alpha=0.59 \pm 0.05$, when expressed in the mass spectrum form $\phi \propto M^{-\alpha}$ ) and compared its slope to the value measure for the Pleiades. This comparison indicates that the two cluster mass functions are, indeed, very similar.
\end{abstract}

Key words. open clusters and associations: individual: Alpha Per - stars: low-mass, brown dwarfs stars: luminosity, mass functions

\section{Introduction}

In an ongoing effort to discover low-mass stars and brown dwarfs (BDs) belonging to young open clusters, we have studied the association Alpha Per. This is a well-known nearby cluster with $(m-M)_{0}=6.23(176 \mathrm{pc})$. The interstellar reddening is also low, $A_{V}=0.30$ (Pinsonneault et al. 1998). The normally quoted age for the cluster, based on isochrone fitting of the upper main sequence (MS), is of order $50 \mathrm{Myr}$ (cf. Meynet et al. 1993), though models with a larger amount of convective core overshoot can yield ages up to about $80 \mathrm{Myr}$ (Ventura et al. 1998). Recently, using the data we published in a preliminary study of the BD population of the cluster (Stauffer et al. 1999), we estimated the age as $90 \mathrm{Myr}$, based on the location of the lithium depletion boundary. The theoretical background to this method can be found in Kumar (1963) and D'Antona \& Mazzitelli (1994), and when applied to the Pleiades (Basri et al. 1996; Rebolo et al. 1996; Stauffer et al. 1998a) and IC 2391 (Barrado y Navascués et al. 1999) has also yielded ages $50 \%$ older than previously assumed. A review of these results may be found in Basri (2000).

Send offprint requests to: D. Barrado y Navascués,

e-mail: barrado@laeff.esa.es

* Based on observations collected with the Kitt Peak National Observatory, USA, and the Hispano-German Observatory of Calar Alto, Spain.
During the last years, different clusters and star forming regions have been studied intensively and their BD population revealed. The Pleiades, Alpha Per cluster, IC 2391, M 35, NGC 2516, Taurus, the Trapezium cluster, Sigma Orionis cluster, Cha I dark cloud, Upper-Sco OB association, and IC348 have been investigated (Rebolo et al. 1995; Festin 1997; Bouvier et al. 1998; Stauffer et al. 1998a,b, 1999; Briceño et al. 1998; Barrado y Navascués et al. 1999, 2001a,b, 2002; Neuhäuser \& Comeron 1999; Zapatero-Osorio et al. 1999, 2000; Lucas \& Roche 2000; Luhman 1999, 2000; Martín et al. 1999, 2000, 2001; Béjar et al. 2001; Pinfield et al. 2000; Ardila et al. 2000; Najita et al. 2000; Moraux et al. 2001; etc.). All these works show that BDs are quite numerous and that the mass function (MF) usually presents an increase for very lowmass objects. In any case, the total mass below the substellar limit only contributes a few percent to the total mass of the parent cluster, at least in the case of the Pleiades ( 3-5\%, Bouvier et al. 1998; Hodgkin \& Jameson 2000). However, it is not clear whether this MF is universal and this result can be extrapolated to other young clusters.

In this paper, we present a new deep, wide-field optical survey of the Alpha Per cluster. We have followed-up the optical candidates in the near-infrared using new infrared imaging data and the 2MASS catalogue (Skrutskie et al. 1997). Using this wealth of data, we have been able to establish the presence of a substantial population of BDs in the cluster, and derived its substellar MF. 
Table 1. Field centers for KPNO 4 m CCD Mosaic Images.

\begin{tabular}{lcc}
\hline \hline Field & RA & DEC \\
\cline { 2 - 3 } & \multicolumn{2}{c}{$(2000.0)$} \\
\hline A & $03: 27: 10.0$ & $49: 24: 00.0$ \\
B & $03: 28: 00.0$ & $48: 42: 00.0$ \\
C & $03: 24: 50.0$ & $48: 42: 00.0$ \\
D & $03: 21: 40.0$ & $48: 42: 00.0$ \\
E & $03: 19: 35.0$ & $49: 27: 00.0$ \\
F & $03: 33: 45.0$ & $49: 35: 00.0$ \\
G & $03: 33: 45.0$ & $49: 04: 00.0$ \\
H & $03: 33: 45.0$ & $48: 34: 00.0$ \\
I & $03: 33: 45.0$ & $50: 06: 00.0$ \\
J & $03: 30: 30.0$ & $50: 09: 00.0$ \\
K & $03: 27: 00.0$ & $50: 13: 00.0$ \\
L & $03: 23: 10.0$ & $48: 11: 00.0$ \\
M & $03: 21: 00.0$ & $50: 48: 00.0$ \\
\hline
\end{tabular}

\section{Observations}

\subsection{Optical survey}

We obtained deep optical imaging of the Alpha Per open cluster using the KPNO 4-m CCD mosaic camera, MOSA, on November 19-22 1998. Field centres were selected in order to cover as much of the central part of the cluster as possible while avoiding the brightest stars in the field (Table 1). All of the images were obtained with the facility $R$ and $I$ filters. Weather during the run was variable: Nov. 19 was generally overcast with periods of only moderate cirrus; Nov. 20 was clear but with extremely poor seeing; Nov. 21 was clear with good seeing; and Nov. 22 was again partly cloudy. In order to provide calibrated photometry for all stars, we overlapped the survey fields so as to provide photometric calibration even for fields whose deep images were obtained through light cirrus. The average seeing for the deep images was of order 1.0 arcsec. For most fields, we obtained two $900 \mathrm{~s} R$ images and two $420 \mathrm{~s} I$ images; a few of the fields just designed to tie together the photometry were only observed with 100 or 200 s exposures. In all, we obtained deep imaging of 13 fields, thus corresponding to a total area on the sky of order 3 square degrees. Photometric calibration was derived from short exposures of three Landolt (1992) fields in SA92, SA98 and SA101. Initial results from analysis of the shorter exposures obtained during this run were reported by Stauffer et al. (1999).

\subsection{Infrared follow-up}

In addition to the KPNO/MOSA observations, we undertook several infrared observing campaigns at the Hispano-German Calar Alto Observatory in order to obtain additional data for candidate cluster members (see Sect. 3.2). These runs included November 22-23 1999, using Omega-Cass (1024² pixels, $0.3 \mathrm{arcsec} / \mathrm{pixel}$ ) on the 3.5-m telescope; February 16-21 2001 and November 6-7 2001, using MAGIC (256 ${ }^{2}$ pixels, 0.64 arcsec/pixel) on the 2.2-m telescope; and December 11-14 2000 and December 28-31 2001 using Omega-Prime (1024 ${ }^{2}$ pixels, $0.4 \mathrm{arcsec} / \mathrm{pixel}$ ) on the $3.5-\mathrm{m}$ telescope. We observed a total
Table 2. Previusly known Alpha Per members from Stauffer et al. (1999). Data from 2MASS survey.

\begin{tabular}{lccc}
\hline \hline Name & $J$ & $H$ & $K \mathrm{~s}$ \\
\hline AP300 & 15.5950 .061 & 14.8590 .074 & 14.5380 .083 \\
AP301 & 15.2330 .061 & 14.5260 .072 & 14.2510 .082 \\
AP302 & 15.5720 .066 & 15.1010 .092 & 14.7580 .102 \\
AP303 & 14.9510 .043 & 14.2780 .049 & 14.1270 .062 \\
AP304 & 16.1020 .107 & 15.4180 .134 & 14.9950 .130 \\
AP305 & 15.7290 .077 & 14.9060 .084 & 14.6750 .100 \\
AP306 & 15.2620 .050 & 14.5220 .063 & 13.9640 .051 \\
AP307 & 14.9480 .043 & 14.2800 .055 & 14.0690 .060 \\
AP308 & 14.6770 .035 & 14.1280 .048 & 13.6130 .049 \\
AP309 & 14.4950 .032 & 13.8820 .044 & 13.5750 .045 \\
AP275 & 15.0310 .046 & 14.4880 .059 & 14.1280 .066 \\
AP310 & 15.5430 .065 & 15.1100 .104 & 14.6220 .085 \\
AP311 & 15.4500 .058 & 14.7090 .069 & 14.3480 .069 \\
AP312 & 16.2740 .118 & 15.4830 .131 & 15.1320 .135 \\
AP313 & 15.5280 .066 & 15.0350 .103 & 14.4250 .081 \\
AP314 & 16.1960 .107 & 15.4330 .128 & 15.1330 .151 \\
AP315 & 15.8940 .089 & 15.2340 .111 & 14.6720 .104 \\
AP318 & 15.0490 .049 & 14.5020 .068 & 14.1080 .071 \\
AP319 & 14.9160 .053 & 14.3780 .063 & 14.0180 .069 \\
AP326 & 16.1920 .108 & 15.5560 .125 & 15.1250 .149 \\
\hline
\end{tabular}

of 53 Alpha Per candidates in the magnitude range $K^{\prime}=13.0$ $19.2 \mathrm{mag}$, equivalent to $I_{\mathrm{c}}=15.7-21.7 \mathrm{mag}$. All were observed in the $K^{\prime}$ filter, while 24 were also measured at $J$, and a handful also at $H$.

We also searched the 2MASS point source catalogue (Skrutskie et al. 1997), in order to extend the infrared data coverage. Unfortunately, the present version of the 2MASS catalogue does not completely cover the Alpha Per cluster, due to gaps between the scans in that part of the sky. Moreover, the 2MASS survey does not go deep enough to detect all faint candidates in our survey. In summary, 47 of our candidates were found in the 2MASS catalogue; 25 additional candidates lie in the non-covered areas; and 21 are too faint to have been detected.

In total, all but two BD candidates derived from the optical KPNO MOSA data also have near-infrared photometry. Table 3 lists all available photometry.

\subsection{Previously known Alpha Per members}

In the following analysis of membership, we have also included previously-discovered photometrically-selected members of the cluster (Stauffer et al. 1999). Some of these also have low- and medium-resolution spectroscopy which supports their membership. We also searched the 2MASS catalogue for infrared photometry for these previously-known members (Table 2), for comparison with our MOSA-selected sample. Note, of course, that Alpha Per cluster contains other brighter and more massive members not analysed here (Stauffer et al. 1986, 1989; Prosser 1992, 1994, 1998). 

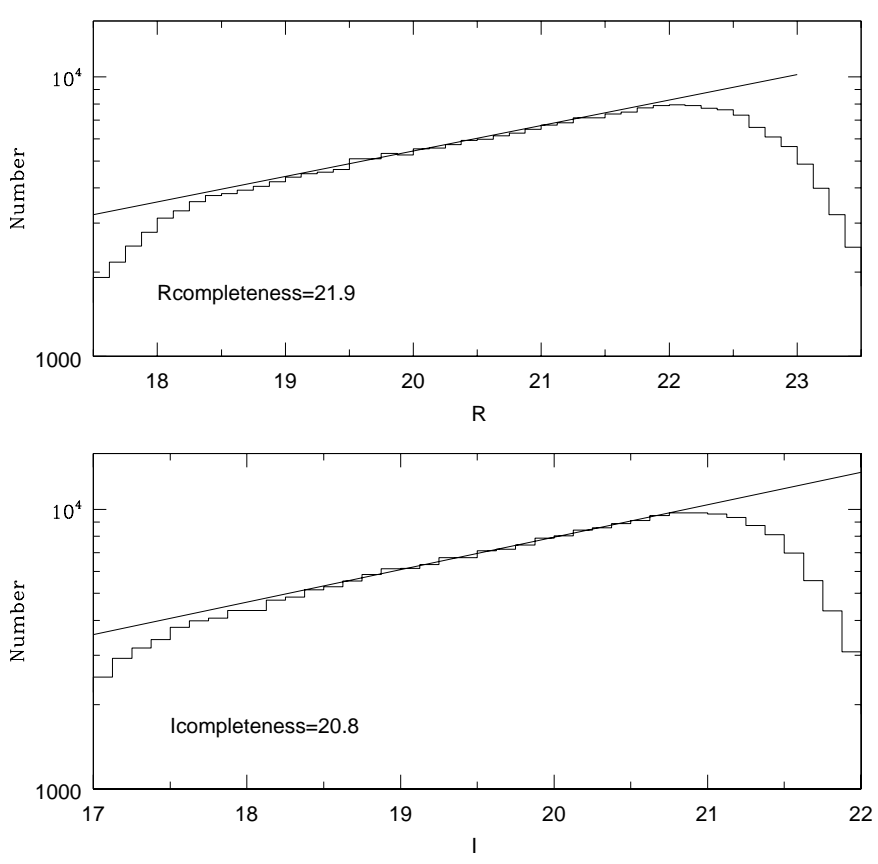

Fig. 1. Completeness of our KPNO/MOSA survey. For candidate members of the Alpha Per cluster, the survey is complete down to $I_{\mathrm{c}}=19.5,(R-I)_{\mathrm{c}}=2.4$.

\section{Analysis}

\subsection{Optical photometry and coordinates}

Raw photometry was derived using PSF-fitting, and calibrated using several standard stars (see Sect. 2.1). We computed the initial coordinates by transforming the $X, Y$ positions of each CCD to $\alpha, \delta$ using field stars with known coordinates. The accuracy of this astrometry is better than 1 arcsec. Following this, we searched for the 2MASS counterparts, and when available, we used the much more accurate 2MASS astrometric positions in Table 3.

Our KPNO/MOSA optical data cover the range $16 \leq I_{\mathrm{c}} \leq$ $22.5,0.0 \leq(R-I)_{\mathrm{c}} \leq 3.5$. Figure 1 allows an estimation of the completeness limits. We have displayed two histograms with the number of detections in the $R_{\mathrm{c}}$ and $I_{\mathrm{c}}$ filters in a logarithmic form. The detections limits are located at the points where the histograms diverge from the straight lines (Wainscoat et al. 1992; Santiago et al. 1996). This is true for a region where field stars dominate the overall population, as in the case of the Alpha Per cluster (many more field stars than cluster members in a particular area). Therefore, for cluster members, we estimate that $I_{\text {complete }} \sim 20.75 \mathrm{mag}, R_{\text {complete }} \sim 21.9$ mag. However, since the $R_{\mathrm{c}}$ filter effectively limits the completeness for cluster members, the actual values are $I_{\text {complete }} \sim 19.5 \mathrm{mag},(R-$ $I)_{\text {complete }} \sim 2.4$. The detection limits reach $I_{\text {limit }} \sim 22.5 \mathrm{mag}$, $R_{\text {limit }} \sim 25.5$ mag.

\subsection{Optical colour-magnitude diagram}

Figure 2 displays all the detections within our MOSA survey area. The straight dashed line denotes the position of a fiducial

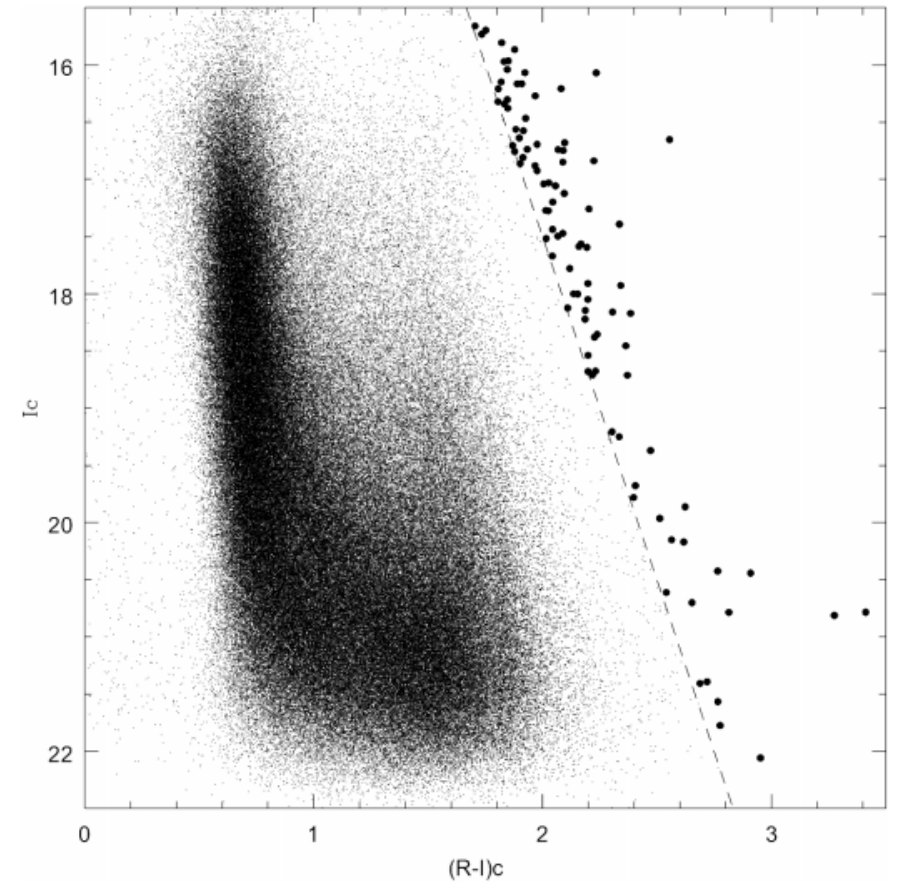

Fig. 2. Colour-Magnitude diagram in the fields we have studied. Solid circles represent the location of our initial selection of cluster candidates. The dashed line corresponds to the fiducial cluster main sequence.

main sequence (i.e., the criterion we have used to select candidate members of the cluster). This line is an empirical zero age main sequence, shifted to fit the locus of previously known Alpha Per members. A total of 260000 stars were detected, by far the majority of them field stars, fairly well separated from the cluster population by a fairly wide gap. This gap is not as sharp however as is seen in the Pleiades (Fig. 2 of Bouvier et al. 1998), and thus we anticipate a stronger contamination of the cluster population in Alpha Per compared to the Pleiades: the latter cluster has a contamination of $~ 30 \%$ (Bouvier et al. 1998; Martín et al. 2000; Moraux et al. 2001).

We have selected 94 candidate members based on the optical CMD. Since some of our fields overlap with each other, some of them have been detected twice, showing similar photometry. The candidate members are listed in Table 3. Figure 3a displays their location in an optical CMD. Bona fide members from Stauffer et al. (1999), based on optical-infrared data and spectroscopy are shown as thick crosses. Thin crosses denote the location of new candidates with no infrared data. Nonmembers, based on the new available data (see next section), appear as asterisks. Finally, possible and probable new members are shown as solid squares and circles, respectively. Also plotted are three $80 \mathrm{Myr}$ isochrones from Baraffe et al. (1998) and Chabrier et al. (2000) (NextGen model as long-dashed line, Dusty model as dotted line, and Cond model as short-dashed line), along with a Leggett (1992) empirical main sequence for the cluster, plotted as a solid line. The $I_{\mathrm{c}}$ magnitudes of Alpha Per members with different masses, derived with a $80 \mathrm{Myr}$ NextGen isochone, are also indicated. 
Table 3. $\alpha$ Per data from KPNO/MOSA 1998. Probable members, possible members, probable non-members, and no IR data.

\begin{tabular}{|c|c|c|c|c|c|c|c|c|c|c|c|}
\hline ID & $\begin{array}{l}\text { alpha delta } \\
\text { (J2000.0) }\end{array}$ & $I_{\mathrm{c}}$ & $(R-I)_{\mathrm{c}}$ & $\delta I \delta R$ & $J$ & $\frac{H}{\text { 2MASS }}$ & $K \mathrm{~s}$ & $J$ & $K^{\prime}$ & Source & Mem. \\
\hline AP282 & $32422.55+482426.1$ & 15.217 & 1.712 & 0.0200 .002 & 13.6900 .030 & 13.0190 .035 & 12.7690 .033 & $\overline{--}$ & $\overline{--}$ & $\overline{-}$ & $\overline{\mathrm{Y}}$ \\
\hline AP283 & $32438.78+481717.1$ & 15.696 & 1.752 & 0.0150 .003 & 14.1380 .030 & 13.5200 .036 & 13.2760 .044 & -- & -- & _- & $\mathrm{Y}$ \\
\hline AP301 & $31809.05+492519.0$ & 17.594 & 2.194 & 0.0040 .005 & 15.2330 .062 & 14.5260 .072 & 14.2510 .082 & 14.9700 .019 & 13.8540 .021 & $\mathrm{~b}$ & $\mathrm{Y}$ \\
\hline AP305 & $31921.60+492331.0$ & 18.355 & 2.239 & 0.0090 .012 & 15.7290 .078 & 14.9060 .084 & 14.6750 .100 & 15.7740 .014 & 14.7030 .018 & b & $\mathrm{Y}$ \\
\hline AP306 & $31941.32+503045.1$ & 18.171 & 2.385 & 0.0030 .008 & 15.2620 .051 & 14.5220 .063 & 13.9640 .051 & -- & -- & - & Y? \\
\hline AP309 & $32240.65+480033.6$ & 16.378 & 1.848 & 0.0030 .002 & 14.4950 .035 & 13.8820 .045 & 13.5750 .046 & -- & -- & - & $\mathrm{Y}$ \\
\hline AP311 & $32308.67+480450.6$ & 17.497 & 2.067 & 0.0040 .005 & 15.4500 .060 & 14.7090 .070 & 14.3480 .070 & -- & -- & - & $\mathrm{Y}$ \\
\hline AP315 & $32634.10+490746.1$ & 18.049 & 2.198 & 0.0090 .012 & 15.8940 .090 & 15.2340 .111 & 14.6720 .104 & 15.8990 .022 & 14.9820 .032 & b & $\mathrm{Y}$ \\
\hline AP327 & $32031.74+493959.6$ & 15.056 & 1.959 & 0.0890 .016 & 13.9170 .035 & 13.3880 .038 & 13.0700 .037 & -- & -- & - & $\mathrm{N}$ \\
\hline AP328 & $32125.61+481001.0$ & 15.193 & 1.720 & 0.0830 .015 & 14.1540 .033 & 13.3970 .037 & 13.1080 .038 & -- & -- & - & $\mathrm{N}$ \\
\hline AP329 & $32356.34+480921.0$ & 15.479 & 1.685 & 0.0260 .002 & 13.9170 .030 & 13.2900 .033 & 13.0350 .035 & -- & -- & - & $\mathrm{Y}$ \\
\hline AP330 & $33415.6+495848$ & 15.659 & 1.705 & 0.0060 .027 & -- & -- & -- & -- & 13.0030 .008 & d & $\mathrm{Y}$ \\
\hline AP331 & $33205.9+500555$ & 15.731 & 1.734 & 0.0330 .042 & -- & -- & -- & -- & 13.2440 .009 & d & $\mathrm{Y}$ \\
\hline AP332 & $32516.90+483609.0$ & 15.805 & 1.821 & 0.0030 .010 & 14.0530 .036 & 13.4100 .040 & 13.2000 .035 & -- & -- & - & $\mathrm{Y}$ \\
\hline AP333 & $32513.55+502733.0$ & 15.865 & 1.878 & 0.0140 .008 & 14.2360 .031 & 13.6030 .034 & 13.3230 .039 & -- & -- & - & $\mathrm{Y}$ \\
\hline AP334 & $32245.47+482133.1$ & 15.965 & 1.850 & 0.0130 .004 & 14.2270 .034 & 13.6410 .041 & 13.3420 .036 & -- & -- & - & $\mathrm{Y}$ \\
\hline AP335 & $32852.96+501925.9$ & 15.970 & 1.832 & 0.0340 .008 & 14.4280 .041 & 13.7610 .040 & 13.4150 .047 & -- & -- & - & $\mathrm{Y}$ \\
\hline AP336 & $33155.3+490831$ & 16.039 & 1.846 & 0.0260 .214 & - & - & -- & -- & 14.6590 .031 & d & $\mathrm{N}$ \\
\hline AP337 & $33407.5+483208$ & 16.067 & 1.923 & 0.0400 .004 & -- & -- & -- & -- & 13.4210 .010 & $\mathrm{~d}$ & $\mathrm{Y}$ \\
\hline AP338 & $32452.65+484612.8$ & 16.069 & 2.235 & 0.0420 .104 & 15.1060 .048 & 14.4810 .060 & 14.4400 .082 & -- & -- & - & $\mathrm{N}$ \\
\hline AP339 & $32633.24+500741.7$ & 16.148 & 1.819 & 0.0430 .003 & 14.3310 .037 & 13.7340 .037 & 13.3750 .037 & -- & -- & - & $\mathrm{Y}$ \\
\hline AP340 & $32134.85+481628.7$ & 16.164 & 1.909 & 0.0020 .002 & 14.4360 .034 & 13.7810 .041 & 13.5470 .043 & -- & -- & - & $\mathrm{Y}$ \\
\hline AP341 & $33103.39+502441.6$ & 16.165 & 1.889 & 0.0080 .002 & 14.4210 .040 & 13.7940 .046 & 13.4430 .047 & -- & -- & - & $\mathrm{Y}$ \\
\hline AP342 & $32539.24+484521.1$ & 16.207 & 2.081 & 0.0110 .068 & 15.0950 .044 & 14.4470 .057 & 14.3770 .075 & -- & -- & - & $\mathrm{N}$ \\
\hline AP343 & $32348.48+483643.0$ & 16.208 & 1.806 & 0.0070 .012 & 14.6190 .045 & 14.0030 .050 & 13.7140 .052 & -- & -- & - & $\mathrm{Y}$ \\
\hline AP344 & $32652.0+500033$ & 16.270 & 1.968 & 0.0220 .002 & -- & -- & -- & -- & 13.4220 .006 & $\mathrm{c}$ & $\mathrm{Y}$ \\
\hline AP345 & $33345.8+500853$ & 16.302 & 1.846 & 0.0050 .008 & -- & -- & -- & 14.4680 .010 & 13.6510 .012 & d & $\mathrm{Y}$ \\
\hline AP346 & $32130.06+484923.2$ & 16.321 & 1.805 & 0.0500 .022 & 14.6210 .037 & 13.9790 .045 & 13.6720 .047 & -- & -- & - & $\mathrm{Y}$ \\
\hline AP347 & $33133.77+495202.1$ & 16.342 & 1.833 & 0.0070 .004 & 14.6010 .045 & 13.9670 .050 & 13.6690 .055 & -- & -- & _- & $\mathrm{Y}$ \\
\hline AP348 & $32648.2+484400$ & 16.466 & 1.926 & 0.0320 .009 & -- & -- & -- & -- & -- & - & - \\
\hline AP349 & $32647.9+500216$ & 16.562 & 1.883 & 0.0110 .002 & -- & -- & -- & -- & 13.7930 .005 & $\mathrm{c}$ & $\mathrm{Y}$ \\
\hline AP350 & $33206.8+492523$ & 16.574 & 1.916 & 0.0240 .009 & -- & -- & -- & -- & 13.9740 .020 & d & Y? \\
\hline AP351 & $32847.8+500201$ & 16.638 & 1.898 & 0.0080 .004 & -- & -- & -- & 14.7270 .033 & 13.9520 .019 & d & $\mathrm{Y}$ \\
\hline AP352 & $31951.98+484822.6$ & 16.652 & 2.555 & 0.0090 .007 & 13.9990 .030 & 13.3440 .035 & 12.9220 .037 & -- & -- & - & $\mathrm{N}$ \\
\hline AP353 & $32448.66+484947.0$ & 16.680 & 2.097 & 0.0040 .005 & 14.6220 .037 & 13.9750 .049 & 13.6190 .051 & -- & -- & - & $\mathrm{Y}$ \\
\hline AP354 & $32731.64+485323.3$ & 16.693 & 1.976 & 0.0090 .024 & 14.6400 .043 & 14.1040 .052 & 13.7250 .056 & -- & -- & - & $\mathrm{Y}$ \\
\hline AP355 & $32233.15+484700.3$ & 16.703 & 1.869 & 0.0090 .005 & 14.8520 .043 & 14.2550 .059 & 13.9790 .058 & -- & -- & - & $\mathrm{Y}$ \\
\hline AP356 & $31822.06+492019.4$ & 16.737 & 1.933 & 0.0090 .004 & 14.7840 .047 & 14.2400 .064 & 14.0680 .064 & -- & -- & - & $\mathrm{N}$ \\
\hline AP357 & $33242.5+495010$ & 16.740 & 2.068 & 0.0150 .004 & -- & -- & -- & -- & -- & - & - \\
\hline AP357 & “" “ & 16.748 & 2.090 & 0.0020 .004 & -- & -- & -- & -- & -- & - & - \\
\hline AP358 & $33437.8+491353$ & 16.756 & 1.878 & 0.0030 .005 & -- & -- & -- & 14.7450 .011 & 14.0580 .021 & d & $\mathrm{Y}$ ? \\
\hline AP359 & $32337.13+483715.3$ & 16.809 & 1.914 & 0.0020 .004 & 14.9480 .045 & 14.2960 .057 & 14.0030 .057 & -- & -- & - & $\mathrm{Y}$ \\
\hline AP360 & $33224.0+501658$ & 16.838 & 2.224 & 0.0020 .013 & -- & -- & -- & 14.6340 .010 & 13.8310 .016 & d & $\mathrm{Y}$ ? \\
\hline AP361 & $32314.03+484221.3$ & 16.849 & 2.089 & 0.0200 .327 & 15.6580 .067 & 15.2720 .106 & 15.2900 .175 & -- & -- & - & $\mathrm{N}$ \\
\hline AP362 & $32524.45+484521.3$ & 16.862 & 1.902 & 0.0080 .070 & 16.0450 .087 & 15.8000 .161 & 15.6360 .225 & -- & -- & - & $\mathrm{N}$ \\
\hline AP363 & $32400.33+475529.7$ & 16.880 & 1.967 & 0.0020 .002 & 14.9990 .046 & 14.2950 .051 & 13.9080 .058 & -- & -- & - & $\mathrm{Y}$ \\
\hline AP364 & $32039.16+493206.0$ & 16.924 & 1.976 & 0.0070 .004 & 14.9330 .052 & 14.4280 .074 & 14.0020 .059 & -- & -- & - & $\mathrm{Y}$ \\
\hline AP365 & $32822.94+491124.0$ & 17.030 & 2.027 & 0.0100 .007 & 15.2330 .057 & 14.4730 .061 & 14.1370 .075 & -- & -- & - & $\mathrm{Y}$ \\
\hline AP366 & $32635.50+491543.8$ & 17.040 & 2.005 & 0.0530 .011 & 15.0870 .048 & 14.4860 .058 & 14.0930 .061 & -- & -- & - & $\mathrm{Y}$ \\
\hline AP367 & $33440.80+500343$ & 17.056 & 2.057 & 0.0020 .004 & -- & -- & -- & 15.0800 .015 & 14.1910 .017 & d & $\mathrm{Y}$ \\
\hline AP368 & $32303.38+485305.8$ & 17.123 & 2.095 & 0.0040 .007 & 15.0310 .048 & 14.4880 .060 & 14.1280 .067 & -- & - - & - & $\mathrm{Y}$ \\
\hline AP368 & “" “ & 17.198 & 2.045 & 0.0100 .011 & “" & "“ " & "“" & -- & -- & _- & $\mathrm{Y}$ \\
\hline AP369 & $32644.9+502509$ & 17.258 & 2.203 & 0.0050 .008 & -- & -- & -- & 15.0710 .011 & 14.0670 .012 & $\mathrm{~d}$ & $\mathrm{Y}$ \\
\hline AP370 & $32421.28+485859.5$ & 17.271 & 2.015 & 0.0030 .007 & 15.3420 .056 & 14.7240 .080 & 14.4200 .081 & -- & -- & - & $\mathrm{Y}$ ? \\
\hline AP371 & $32359.92+485410.7$ & 17.274 & 2.026 & 0.0020 .009 & 15.4220 .063 & 14.7240 .077 & 14.4480 .086 & -- & -- & - & $\mathrm{Y}$ ? \\
\hline AP372 & $32802.20+484107.2$ & 17.391 & 2.336 & 0.0100 .010 & 15.0130 .050 & 14.4650 .054 & 14.0280 .058 & -- & -- & - & $\mathrm{Y}$ \\
\hline AP373 & $33320.6+484549$ & 17.437 & 2.044 & 0.0070 .016 & - & -- & -- & -- & 14.4690 .021 & d & $\mathrm{Y}$ ? \\
\hline AP374 & $33218.8+493218$ & 17.472 & 2.089 & 0.0040 .006 & -- & -- & -- & -- & 14.5540 .022 & d & $\mathrm{Y} ?$ \\
\hline AP375 & $32043.49+505939.6$ & 17.518 & 2.016 & 0.0070 .014 & 15.6470 .069 & 15.2230 .121 & 14.5990 .095 & 15.2250 .025 & 14.2630 .031 & b & $\mathrm{N}$ \\
\hline AP376 & $33243.7+501825$ & 17.564 & 2.168 & 0.0050 .015 & -- & -- & -- & 15.2300 .019 & 14.3380 .029 & $\mathrm{~b}$ & $\mathrm{Y}$ \\
\hline AP377 & $32731.29+483923.3$ & 17.586 & 2.159 & 0.0150 .019 & 15.0940 .055 & 14.4820 .071 & 14.1160 .071 & 14.8960 .007 & 13.9050 .018 & b & $\mathrm{Y}$ \\
\hline AP378 & $32700.3+491438$ & 17.670 & 2.043 & 0.0100 .010 & -- & -- & -- & 15.3740 .025 & 14.4020 .032 & b & $\mathrm{Y}$ \\
\hline AP379 & $32918.74+502210.9$ & 17.779 & 2.119 & 0.0050 .009 & 15.5370 .071 & 15.0850 .088 & 14.4490 .079 & 15.4560 .025 & 14.6720 .040 & b & $\mathrm{Y}$ \\
\hline AP380 & $32503.79+484958.6$ & 17.910 & 2.198 & 0.0040 .008 & 15.6970 .072 & 15.0780 .100 & 14.6250 .102 & 15.3740 .025 & 14.3550 .030 & b & $\mathrm{Y}$ \\
\hline AP381 & $32806.3+484513$ & 17.927 & 2.342 & 0.0090 .013 & $>16.1-$ & $>15.6-$ & $>15.3-$ & 16.2140 .045 & 15.1390 .062 & b & $\mathrm{N}$ \\
\hline AP382 & $33348.08+485228$ & 18.000 & 2.136 & 0.0050 .008 & -- & -- & -- & 15.9260 .029 & 14.9500 .030 & b & $\mathrm{N}$ \\
\hline AP383 & $33222.8+485411$ & 18.003 & 2.154 & 0.0030 .005 & -- & -- & -- & 15.8700 .023 & 15.0820 .039 & b & $\mathrm{N}$ \\
\hline AP384 & $32740.03+483355.8$ & 18.125 & 2.110 & 0.0210 .031 & 15.6890 .081 & 15.1590 .118 & 14.7060 .102 & 15.7930 .026 & 14.8200 .028 & b & $\mathrm{Y}$ \\
\hline AP385 & $32007.42+503953.3$ & 18.145 & 2.187 & 0.0040 .007 & 15.7790 .085 & 15.1280 .108 & 14.7610 .114 & 15.9540 .025 & 15.0030 .031 & b & $\mathrm{Y}$ \\
\hline AP386 & $32202.3+475838$ & 18.160 & 2.306 & 0.0030 .008 & $>16.1-$ & $>15.6-$ & $>15.3-$ & 16.5680 .046 & 15.4620 .053 & b & $\mathrm{N}$ \\
\hline AP387 & $31819.32+491435.1$ & 18.222 & 2.186 & 0.0040 .007 & 15.9270 .106 & 15.2930 .129 & 15.1400 .162 & 15.9550 .022 & 15.0150 .031 & b & $\mathrm{N}$ \\
\hline AP388 & $33147.4+500325$ & 18.380 & 2.227 & 0.0050 .008 & -- & -- & -- & -- & 15.3370 .040 & d & $\mathrm{N}$ \\
\hline AP388 & "“" & " & "“ & “" " & -- & -- & -- & -- & 15.4160 .084 & $\mathrm{e}$ & $\mathrm{N}$ \\
\hline AP389 & $32741.21+484033.9$ & 18.455 & 2.364 & 0.0190 .022 & 15.3390 .067 & 14.6470 .073 & 14.1790 .074 & -- & -- & - & Y? \\
\hline AP390 & $32154.45+483314.9$ & 18.538 & 2.199 & 0.0060 .009 & 16.2160 .295 & 15.5310 .151 & 14.9830 .172 & -- & -- & - & $\mathrm{Y}$ \\
\hline AP391 & $33248.1+495226$ & 18.675 & 2.232 & 0.0120 .014 & -- & -- & -- & -- & 15.5060 .050 & d & $\mathrm{N}$ \\
\hline AP391 & "“" & 18.710 & 2.218 & 0.0050 .008 & -- & -- & -- & -- & "“ “ & “ & $\mathrm{N}$ \\
\hline AP392 & $32827.1+502002$ & 19.207 & 2.304 & 0.0060 .014 & $>16.1-$ & $>15.6-$ & $>15.3-$ & -- & 15.4490 .017 & a & $\mathrm{Y}$ \\
\hline AP393 & $32908.2+482535$ & 19.250 & 2.335 & 0.0220 .035 & $>16.1-$ & $>15.6-$ & $>15.3-$ & -- & 15.7800 .021 & $\mathrm{e}$ & $\mathrm{Y}$ ? \\
\hline AP393 & "“" & " & " & “" “ & " - & "- & "- & -- & 15.8160 .080 & d & $\mathrm{Y}$ ? \\
\hline AP394 & $33526.0+494655$ & 19.370 & 2.472 & 0.0080 .017 & -- & -- & -- & -- & 15.7050 .023 & $\mathrm{a}$ & $\mathrm{Y}$ \\
\hline AP395 & $32444.5+482129$ & 19.678 & 2.406 & 0.0240 .036 & $>16.1-$ & $>15.6-$ & $>15.3-$ & -- & 15.5770 .023 & $\mathrm{a}$ & $\mathrm{Y}$ \\
\hline AP396 & $32816.0+483740$ & 19.781 & 2.398 & 0.0160 .048 & $>16.1-$ & $>15.6-$ & $>15.3-$ & -- & 15.3700 .015 & $\mathrm{a}$ & $\mathrm{Y}$ \\
\hline AP397 & $32121.8+480223$ & 19.862 & 2.623 & 0.0120 .038 & $>16.1-$ & $>15.6-$ & $>15.3-$ & -- & 16.0780 .029 & a & $\mathrm{Y}$ \\
\hline AP398 & $32238.0+485803$ & 19.964 & 2.512 & 0.0110 .028 & $>16.1-$ & $>15.6-$ & $>15.3-$ & -- & 15.8550 .023 & $\mathrm{a}$ & $\mathrm{Y}$ \\
\hline AP399 & $32548.5+500059$ & 20.151 & 2.564 & 0.0140 .045 & $>16.1-$ & $>15.6-$ & $>15.3-$ & -- & 16.3090 .047 & a & $\mathrm{Y}$ \\
\hline
\end{tabular}


Table 3. continued.

\begin{tabular}{|c|c|c|c|c|c|c|c|c|c|c|c|}
\hline ID & $\begin{array}{c}\text { alpha delta } \\
\text { (J2000.0) }\end{array}$ & $I_{\mathrm{c}}$ & $(R-I)_{\mathrm{c}}$ & $\delta I \delta R$ & $J$ & $\frac{H}{\text { 2MASS }}$ & $K \mathrm{~s}$ & $J$ & $K^{\prime}$ & Source & Mem. \\
\hline AP400 & $32848.0+502042$ & 20.170 & 2.617 & 0.0190 .085 & $>16.1-$ & $>15.6-$ & $>15.3-$ & $\overline{--}$ & 16.7110 .175 & $\mathrm{~d}$ & $\bar{N}$ \\
\hline AP400 & "“ & “ & " & " “ & "- & "- & " & -- & 16.8170 .090 & $\mathrm{e}$ & $\mathrm{N}$ \\
\hline AP401 & $32343.5+482239$ & 20.424 & 2.765 & 0.0200 .292 & $>16.1-$ & $>15.6$ & $>15.3-$ & -- & 18.2040 .140 & a & $\mathrm{N}$ \\
\hline AP402 & $33038.7+501639$ & 20.442 & 2.909 & 0.0200 .257 & $>16.1-$ & $>15.6$ & $>15.3-$ & -- & 17.2510 .064 & $\mathrm{e}$ & $\mathrm{N}$ \\
\hline AP403 & " “ & “ & " & "“ & -- & - & -- & -- & 16.9260 .185 & d & Y \\
\hline AP403 & $33204.6+492048$ & 20.611 & 2.541 & 0.0200 .053 & -- & -- & -- & -- & 16.620 .08 & $\mathrm{e}$ & $\mathrm{Y}$ \\
\hline AP404 & $32348.3+482903$ & 20.700 & 2.653 & 0.0300 .125 & $>16.1-$ & $>15.6-$ & $>15.3-$ & -- & 17.9670 .112 & $\mathrm{a}$ & $\mathrm{N}$ \\
\hline AP405 & $32913.0+500524$ & 20.784 & 3.412 & 0.0640 .224 & $>16.1-$ & $>15.6-$ & $>15.3-$ & -- & $>18.4-$ & $\mathrm{e}$ & $\mathrm{N}$ \\
\hline AP406 & $32309.9+481630$ & 20.785 & 2.814 & 0.0250 .127 & $>16.1-$ & $>15.6-$ & $>15.3-$ & -- & 16.2830 .028 & a & Y \\
\hline AP407 & $32359.3+482830$ & 20.811 & 3.275 & 0.0380 .258 & $>16.1-$ & $>15.6-$ & $>15.3-$ & -- & $>18.20-$ & $\mathrm{e}$ & $\mathrm{N}$ \\
\hline AP408 & $32636.0+493658$ & 21.392 & 2.719 & 0.0430 .258 & $>16.1-$ & $>15.6-$ & $>15.3-$ & -- & $>18.02-$ & $\mathrm{e}$ & $\mathrm{N}$ \\
\hline AP409 & $32017.7+504603$ & 21.406 & 2.688 & 0.0450 .153 & $>16.1-$ & $>15.3-$ & $>15.3-$ & 18.7230 .266 & 17.8270 .164 & $\mathrm{a}$ & $\mathrm{Y}$ ? \\
\hline AP410 & $32621.6+484450$ & 21.565 & 2.766 & 0.0600 .519 & $>16.1-$ & $>15.6$ & $>15.3-$ & $>18.37$ & $>17.5-$ & $\mathrm{a}$ & $\mathrm{Y}$ ? \\
\hline AP411 & $32610.1+493753$ & 21.774 & 2.776 & 0.0450 .239 & $>16.1-$ & $>15.6-$ & $>15.3-$ & -- & 19.1380 .451 & $\mathrm{e}$ & $\mathrm{N}$ \\
\hline AP412 & $32603.1+493029$ & 22.057 & 2.952 & 0.0710 .342 & $>16.1-$ & $>15.6$ & $>15.3-$ & -- & $>18.60-$ & $\mathrm{e}$ & $\mathrm{N}$ \\
\hline
\end{tabular}

a) 1999 November, b) 2000 February, c) 2000 December, d) 2001 November, e) 2001 December.

$H$ data from CAHA: Ap345 $H=13.920 \pm 0.008, \mathrm{AP} 351 H=14.203 \pm 0.043, \mathrm{AP} 358 H=14.205 \pm 0.014, \mathrm{AP} 360 H=14.084 \pm 0.011, \mathrm{AP} 367 H=14.448 \pm 0.013, \mathrm{AP} 369 H=14.324 \pm 0.010$.

Note that all colour-magnitude and colour-colour diagrams presented in this paper (this and next subsections) have made use of the following reddening values: $A_{I}=0.179, A_{J}=$ $0.085, A_{K}=0.034$, and $E\left(V-I_{\mathrm{c}}\right)=0.121, E(R-I)_{\mathrm{c}}=0.067$, $E\left(I_{\mathrm{c}}-J\right)=0.094, E\left(I_{\mathrm{c}}-K\right)=0.145$, and $E(J-K)=0.051$. These values were derived from $A_{V}=0.30, R_{V}=3.12$ and the interstellar extinction law and transformation equation between filters and systems published by Rieke \& Lebofsky (1985) and Taylor (1986).

\subsection{Infrared data and membership selection}

Alpha Per candidate members brighter than $I=18.5$ are expected to be detected by the 2MASS survey (Skrutskie et al. 1997), which reaches about $K \sim 15$. We have searched in this database and extracted the IR photometry for them. However, 2MASS has several patches in the Alpha Per area, with gaps between the images and some of our candidates were not observed. For objects at the faint end of 2MASS and fainter than it, we have carried out our own deep IR observations. All these data are listed in Table 3.

Figures 3b-d show optical/IR-colour magnitude diagrams for the candidates derived from the KPNO, Calar Alto, and 2MASS data (Table 3). Symbols are as in Fig. 3a (see key or Sect. 3.2). As in the case of panel a, a reddening vector for $A_{V}=2.0$ has been included for comparison purposes. Note that neither of the two isochrones fully agree either with the new MOSA data or with the previously discovered bona fide members (Stauffer et al. 1999). This is perhaps not unexpected, as even these models which include state-of-the-art model atmospheres do not as yet accurately reproduce the optical SED's for late type stars and hence, in particular, the $R$ band magnitudes are only approximate for the very cool objects in our figures. Figure 4 shows an optical-infrared colour-colour diagram for the same sample. The solid line corresponds to an empirical main sequence based on Leggett (1992) data. Objects with full agreement between their photometric locations in the four colour-magnitude diagrams and those expected for bona fide cluster members are henceforth classified as probable cluster members. Others, with slight disagreements in their photometry are classified as possible members. Finally, some (shown as asterisks) could be readily rejected as cluster members. The classification of each object is shown in the last column of Table 3. Out of the initial 94 candidates, more than half (54) have been classified as probably cluster members and 12 as possibles, while 26 objects turn out to be non-members. The remaining 2 candidates do not have infrared photometry and cannot be classified. Thus, the contamination of the sample lies in the range $30-45 \%$, similar or larger than in the case of the Pleiades (Bouvier et al. 1998; Martín et al. 2000; Moraux et al. 2001).

\subsection{Luminosity and mass functions}

\subsubsection{Luminosity function}

We have computed the cluster luminosity function (LF) at the end of the main sequence. We have done this both before and after rejecting the non-members from our initial membership list. The LF is illustrated in Fig. 5, where we represent the histograms computed with the initial and final candidate members as solid circles (solid and dashed lines, respectively) and a control group of objects selected in the same fashion, but with colours bluer than them, with an arbitrary shift of $\Delta(R-I)=-0.2 \mathrm{mag}$. Based on this selection criterion, the vast majority of the comparison sample should be field stars, at a distance similar to that of the cluster stars. The dotted segment represents the location of the completeness limit, which corresponds to $\sim 0.05 M_{\odot}$. Poisson noise for each value is represented with the vertical error bar. Note that apparent $I_{\mathrm{c}}$ magnitudes are displayed at the bottom $x$-axis, whereas absolute magnitudes are represented at the top $x$-axis. The Alpha Per cluster LF presents two peaks (at this magnitude range): one at $M\left(I_{\mathrm{c}}\right) \sim 10$ and another at $14.5 \mathrm{mag}$. In the first case, the peak appears both in the Alpha Per member list and in the control group described above. In the second case, the sample is not complete, and it could be strongly influenced by pollution by spurious members, as the comparison with the control group suggests. Note that the peak at $M\left(I_{\mathrm{c}}\right) \sim 10$ is present in some other young clusters, including the Pleiades (Zapatero Osorio 1997), but not all, for example, NGC 2516 (Jeffries et al. 2001; Barrado y Navascués et al. 2002), a rich, 125 Myr old cluster 

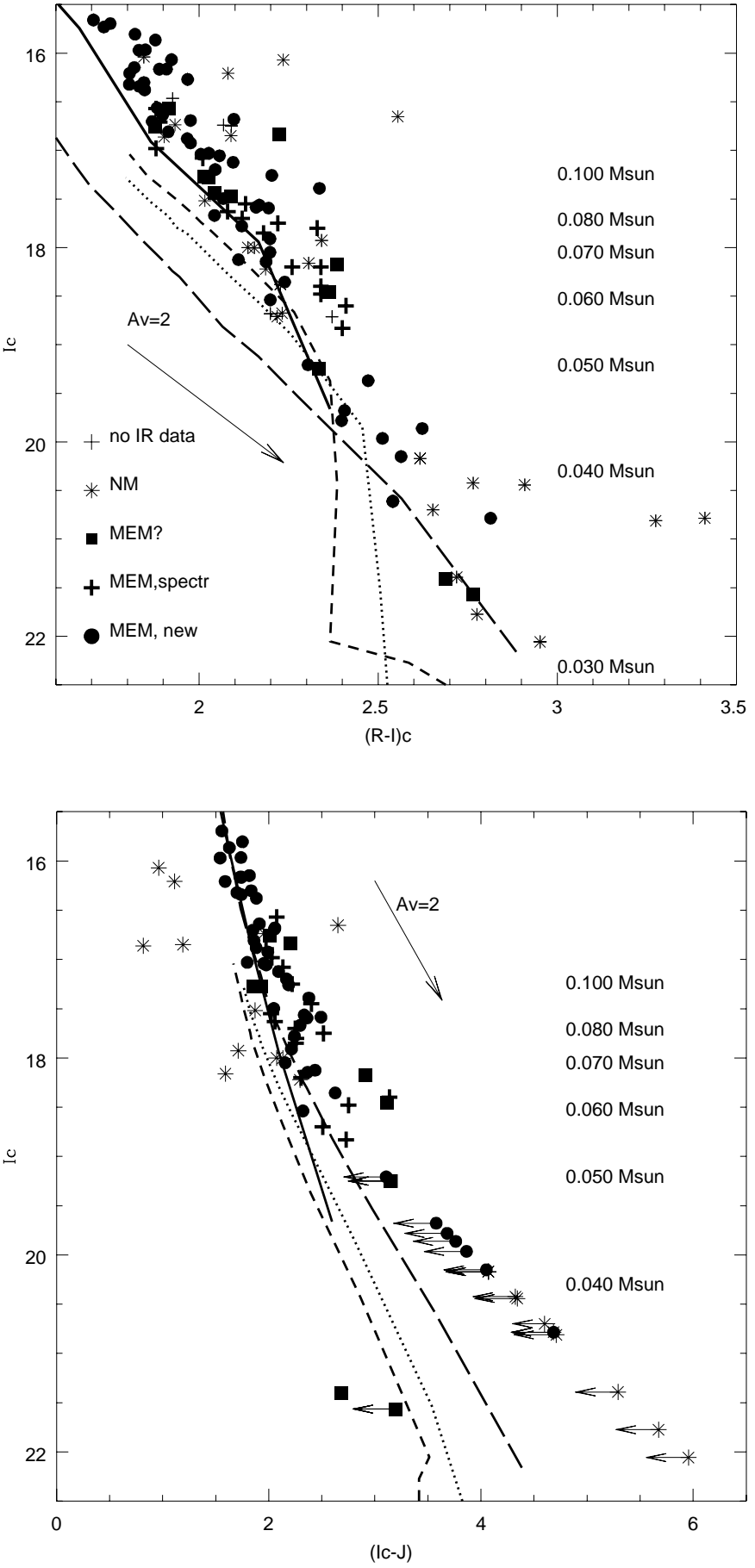

Fig. 3. Colour-Magnitude diagrams of Alpha Per cluster. See key for the meaning of the symbols. Three $80 \mathrm{Myr}$ isochrones from Baraffe et al. (1998) and Chabrier et al. (2000) (NextGen model as longdashed lines, Dusty model as dotted lines, and Cond model as shortdashed lines) are displayed together with a Leggett (1992) main sequence (solid lines). For several $I_{\mathrm{c}}$ magnitudes, the masses of cluster members are indicated in the right-hand side of each panel. We have included a reddening vector for $A_{V}=2$ for comparison purposes.

that is either slightly metal deficient (Jeffries et al. 1997, 1998, 2001) or approximately solar metallicity (Debernardi \& North 2001; Terndrup et al. 2002). The peak in the LF for NGC 2516 appears to occur about a magnitude fainter than for the Pleiades and Alpha Per (that is, at about $M\left(I_{\mathrm{c}}\right) \sim 11$ ). Another simi-
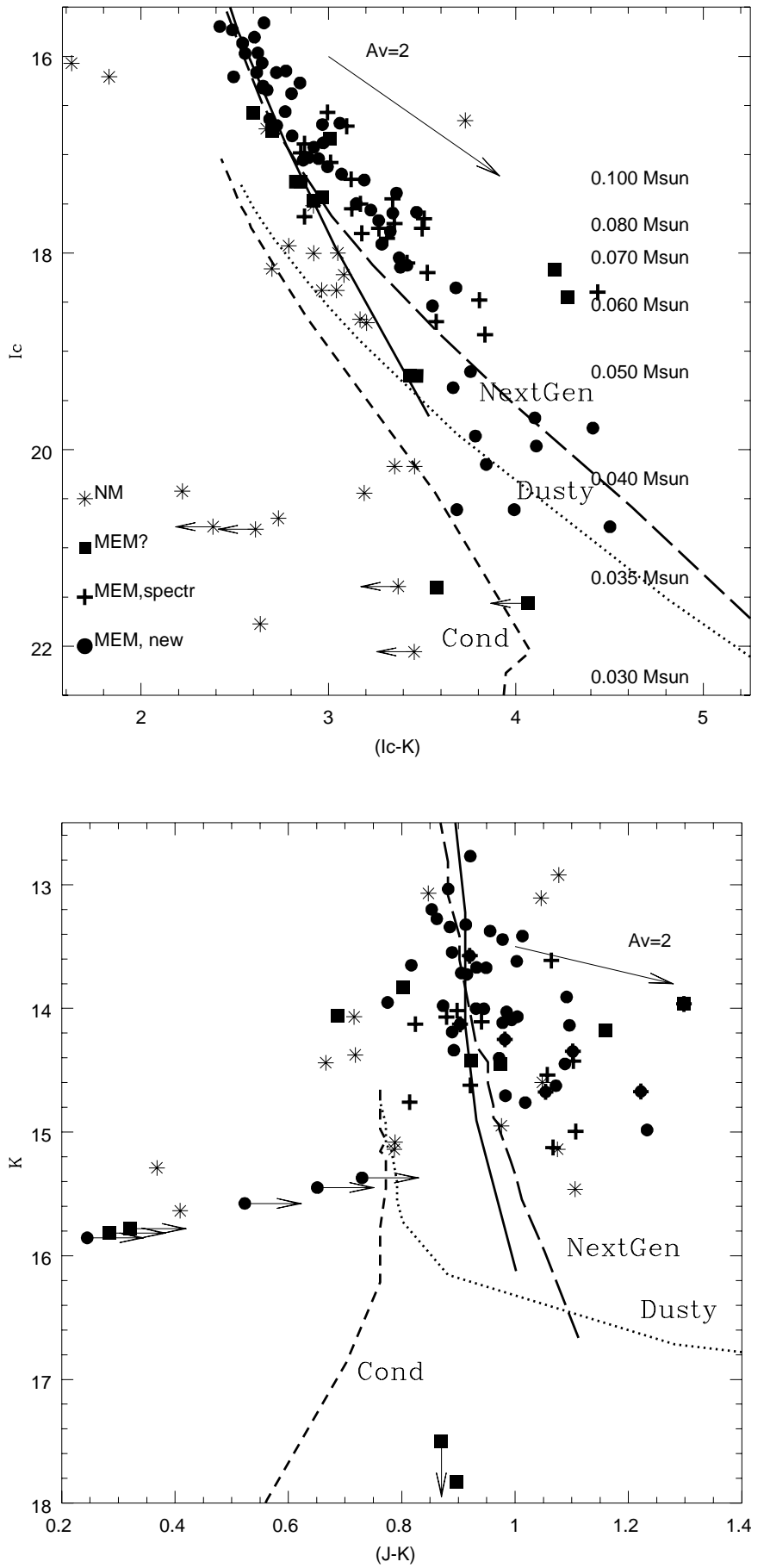

Fig. 3. continued.

lar age cluster, M 35, appears to have the peak occur at about $M\left(I_{\mathrm{c}}\right) \sim 9$ (Barrado y Navascues et al. 2001a). Therefore, the exact absolute magnitude of the peak does not seem to be universal, at least for 100-200 Myr clusters.

The gap in the Alpha Per LF at $M\left(I_{\mathrm{c}}\right) \sim 12.5$ (corresponding to M6-M8 spectral type or about $0.055 M_{\odot}$ for an age of $80 \mathrm{Myr}$ ) has been also found in other clusters. This is the case of the young cluster IC 2391 (Barrado y Navascués et al. 2001b). Jameson (2002) noticed that this gap, which appears at about M 7 spectral type, is present in clusters with ages ranging from few million years (Sigma Orionis cluster), up to at least 


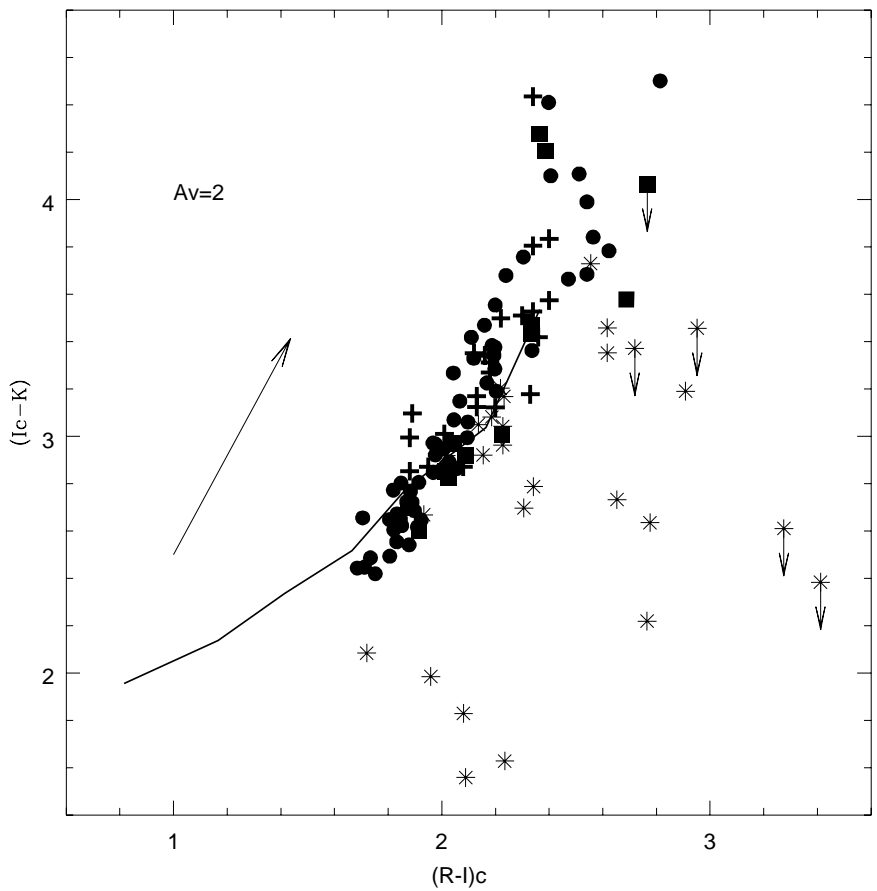

Fig. 4. Optical-IR Colour-Colour Diagram for Alpha Per cluster. Symbols as in Fig. 3. The solid line comes from Leggett (1992).

seven hundred million year (Praesepe). It is clearly seen in the Pleiades too. They suggested that it could be related to the formation at these temperatures of large dust grains, although it might be related to some specific behavior in the luminositymass relationship or to the formation mechanism of these low mass objects (the efficiency of the fragmentation and collapse for cores with this mass range within the parental molecular cloud).

\subsubsection{Mass function}

We have computed the Mass Function of the Alpha Per cluster using the non-dusty (Next-Gen) models by Baraffe et al. (1998) Note that the global behavior of the MF is not strongly affected by the particular set of isochrones, as we have shown in the case of M 35 (Barrado y Navascués et al. 2001a) and NGC 2516 (Barrado y Navascués et al. 2002), using evolutionary tracks from Baraffe et al. (1998), D’ Antona \& Mazzitelli (1994, 1997) and Siess et al. (2000). In order to assess the effects of contamination by field stars in our survey, and biases due to incomplete coverage in the infrared, we have estimated the MF based on several different subsamples of the candidate list. For the first sample, we used the full candidate list derived from the optical data only. In the second, we removed definite non-members. In the third, we removed sources without infrared photometry. Finally, in the fourth, we included only those sources classified as probable cluster members. All four derived MFs are shown in Fig. 6 along with the MF for the Pleiades (Bouvier et al. 1998; Moraux et al. 2001; Zapatero Osorio et al. 2002).

Overall, the behaviour of the Alpha Per cluster MF is very similar to that of the Pleiades, both in the overlapping mass range and for less massive brown dwarfs. However, the MF

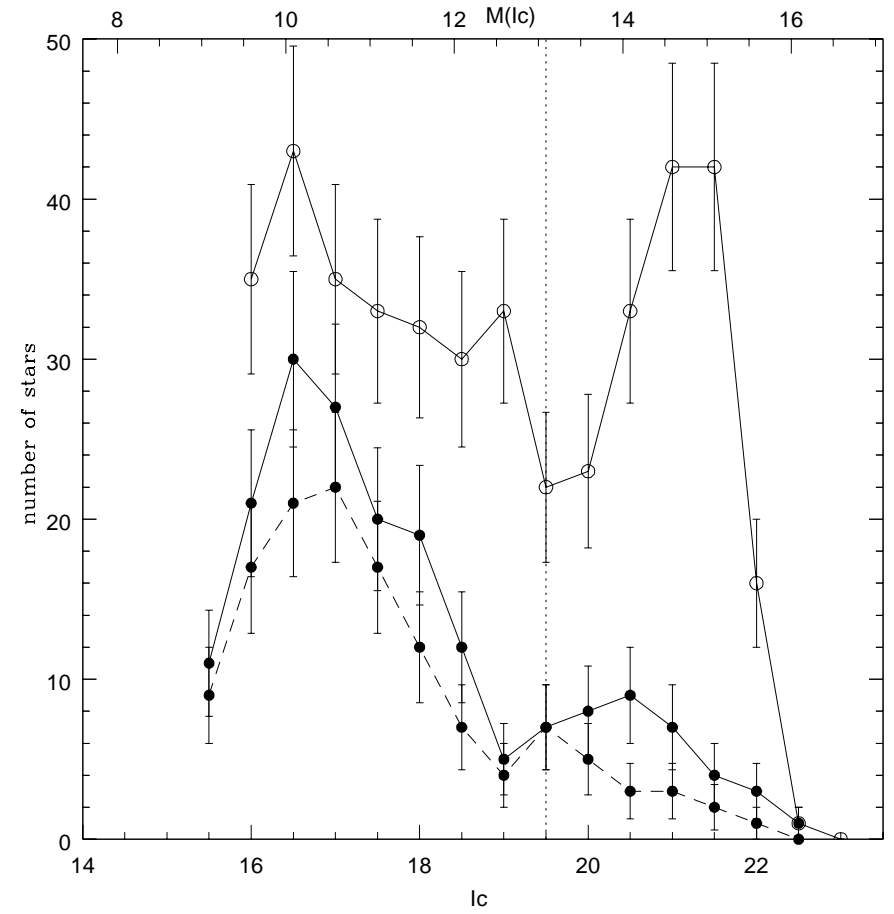

Fig. 5. Luminosity Function of Alpha Per cluster. The lower LFs correspond (solid circles) to the initial MOSA candidate members of the cluster, whereas the upper curve (open circles) was derived for a comparison sample of field stars. In the first case, we show the LF before and after rejecting the non-members (solid and dashed lines, respectively).

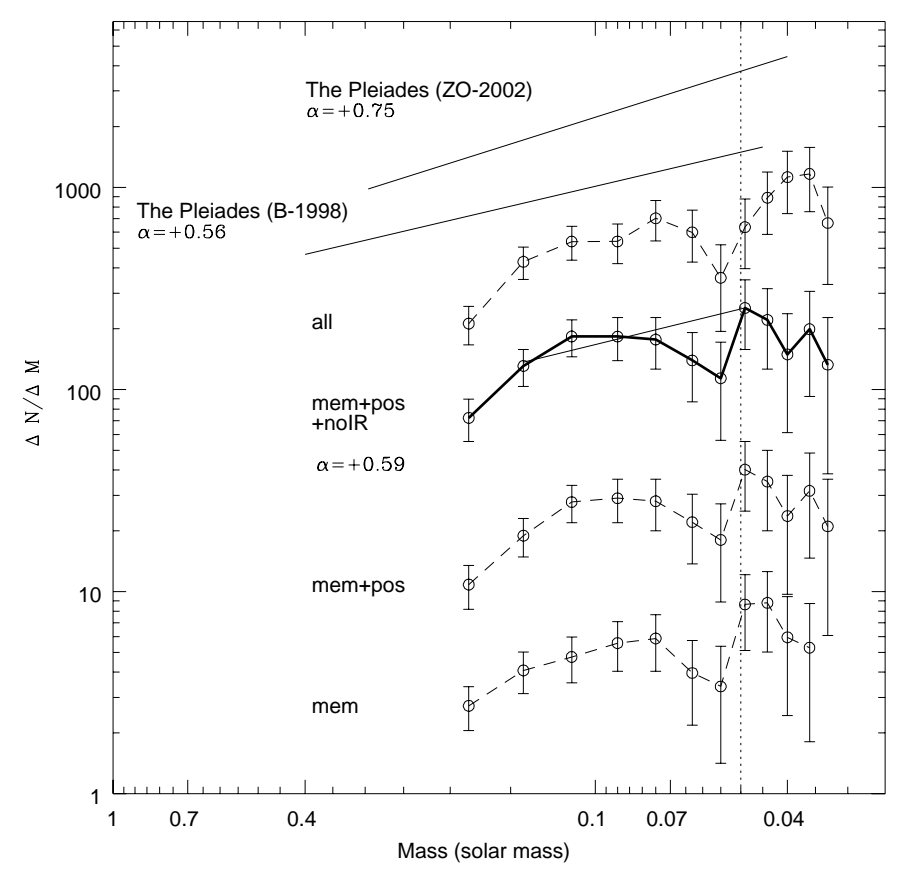

Fig. 6. Mass functions for the Alpha Persei cluster, illustrating the effect of contamination by field stars. These MFs were derived using a $80 \mathrm{Myr}$ isochrone (Baraffe et al. 1998). The most probable MF is shown as a thick line. A linear fit for this MF is also included, with a power law index of $\alpha=0.59 \pm 0.05$. For comparison, we have included the Pleiades MF by Bouvier et al. (1998), valid between 0.40 and $0.045 M_{\odot}$ and by Zapatero Osorio et al. (2002), which was derived for the mass range $0.3-0.040 M_{\odot}$. 

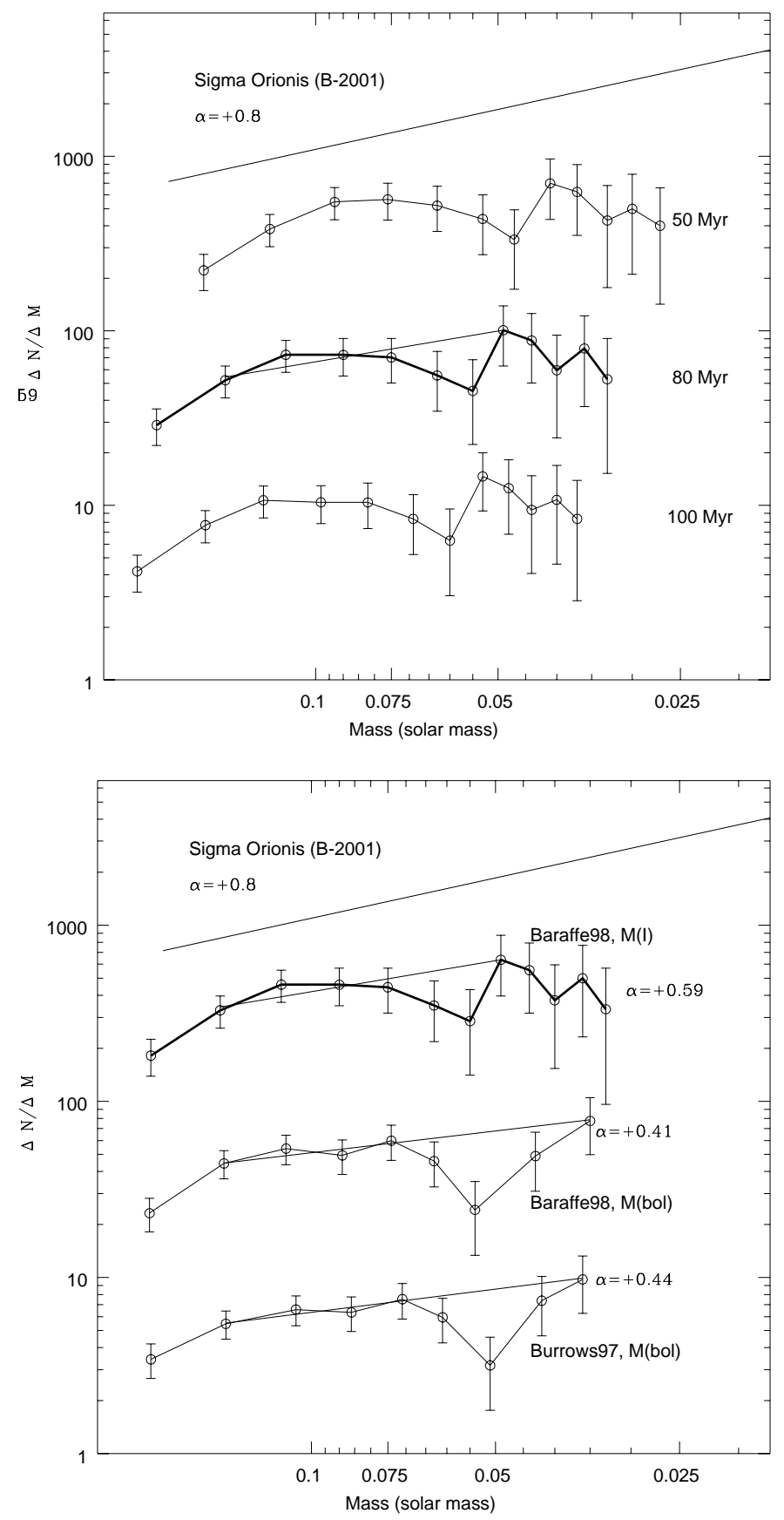

Fig. 7. Mass functions of Alpha Persei, illustrating the effect of the assumed age (panel a) and the choice of theoretical models (panel b). A power law fit and its slope is included in each case. A MF for the Sigma Orionis cluster (Béjar et al. 2001) is shown for comparison purposes (solid segment).

of the Alpha Per cluster shows some possible structure, with a dip about $0.055 M_{\odot}$ and a drop beyond $0.035 M_{\odot}$, regardless the cluster age in the range 50-100 Myr (see next paragraph). Unfortunately, the uncertainties are large, the completeness limit of our MOSA survey is about $0.050 M_{\odot}$, and there is significant pollution by field stars below this mass (peaking at about $\left.0.030 M_{\odot}\right)$.

Figure 7 shows several realisations of the Alpha Per MF as a function of age $(50,80$, and $100 \mathrm{Myr})$, using the complete list of candidates minus the definite non-members (Sect. 3.2).
MFs have been derived for both the non-dusty Baraffe et al. (1998) and Burrows et al. (1997). Masses were derived either from the $M\left(I_{\mathrm{c}}\right)$ magnitude (Baraffe et al. 1998 list these values) or from the bolometric luminosities (after Monet et al. 1992). It is readily seen that the slope, within the uncertainties, and the general shape of the cluster MF is essentially independent of the adopted age or the model. The exact mass of the dip in the MF (assuming it is real), however, does shift slightly depending on the age.

When defining the MF as $\phi \propto M^{-\alpha}$ (or more properly, the mass spectrum), the spectral index $\alpha$ indicates the slope in the diagram we show in Fig. 6. We have fit a power law function to this MF, obtaining $\alpha=0.59 \pm 0.05$. For the Pleiades, this index is 0.6, as derived by Bouvier et al. (1998). See also Martín et al. (2000); Hodgkin \& Jameson (2000); Moraux et al. (2001); Zapatero Osorio et al. (2002) $-\alpha=0.75$; and references therein. Two of the Pleiades MFs are shown in Fig. 6. Béjar et al. (2001) have derived a MF for the younger cluster Sigma Orionis, about $5 \mathrm{Myr}$, down to the planetary mass domain at $\sim 0.013 M_{\odot}$. This MF is shown in Fig. 7. The spectral index of Sigma Orionis cluster $(\alpha=0.8 \pm 0.4)$ also agrees quite well, within the uncertainties, with both Alpha Per cluster and the Pleiades. Note that the Alpha Per data corresponding to the gap at $0.055 M_{\odot}$ have not been included in this fit. In any case, independently of the power law index of the MF, Figs. 6 and 7 clearly show that the MF keeps rising well below the substellar limit for Alpha Per cluster.

\subsection{Brown dwarfs in the cluster}

Recently, both Stauffer et al. (1999) and Basri \& Martín (1999) searched for evidence of BDs in the Alpha Per cluster. Previously, Rebolo et al. (1992) and Zapatero Osorio et al. (1996) tried, without success, to find them in this association. Stauffer et al. (1999) included both low and medium resolution spectroscopy, and some infrared photometry, establishing the location of the lithium depletion boundary (with the lithium age of the cluster, $90 \pm 10 \mathrm{Myr}$ ) and the member list of bona fide members (based on rough radial velocity, lithium detection, $\mathrm{H} \alpha$ emission, spectral types, etc.). Since their spectroscopic data go down to $I_{\mathrm{c}}=18.7$, and the stellar/substellar boundary is located at $I_{\mathrm{c}}=18.2$ (Baraffe et al. 1998), they were able to catalogue 2 objects as probable brown dwarfs (another 3, which were catalogued as possible members, could also be BDs). In our new MOSA sample, there are 27 objects below the substellar frontier. Out of these 27 objects, 11 have infrared photometry which indicates that they are probable members of the cluster and, therefore, brown dwarfs. Another 5 have been classified as possible members and could be BDs if their membership is confirmed. The minimum mass is $0.035 M_{\odot}$. Therefore, it seems likely that we have discovered a substantial population of BDs in the Alpha Per cluster. Note that it is difficult to use the proper motion method to establish Alpha Per membership, since its proper motion (22.93 mas/yr in $\alpha$ and $-25.56 \mathrm{mas} / \mathrm{yr}$ in $\delta$, Robichon et al. 1999), is large enough to be measured, but is also shared by a large fraction of the field stars (see, e.g., Prosser 1992). Therefore, only additional spectroscopic data 
(or better, a combination of optical and infrared photometry, proper motions and spectroscopy) will demonstrate beyond any reasonable doubt that the faint objects listed in our sample are, indeed, brown dwarfs.

\section{Summary}

Optical and near infrared photometry have been used to select a list of probable and possible low-mass members belonging to the young cluster Alpha Per, unveiling a large population of brown dwarf candidates. This information has been used to derive the luminosity and mass function of the cluster in the substellar domain. The index of the mass function, $\alpha=0.59$, is very similar to that determined for the Pleiades (120 Myr) and $\sigma$ Orionis (2-8 Myr) clusters.

Acknowledgements. DBN and JRS acknowledge the hospitality of the Observatoire de Grenoble during the preparation of this paper. We thank D. Montes for his help collecting part of the IR data and M.R. Zapatero Osorio for her comments on an early version of this paper. JB, NL, and MJM acknowledge support from the EC Research Training Network "The Formation and Evolution of Young Stellar Clusters" (HPRN-CT-2000-00155). This work has been partially financed by Spanish "Programa Ramón y Cajal" and AYA2001-1124$\mathrm{CO} 2$ programs.

\section{References}

Ardila, D. R., Martín, E. L., \& Basri, G. 2000, AJ, 120, 479

Baraffe, I., Chabrier, G., Allard, F., \& Hauschildt, P. H. 1998, A\&A, 337,403

Barrado y Navascués, D., Stauffer, J. R., \& Patten, B. M. 1999, ApJ, 522, L56

Barrado y Navascués, D., Stauffer, J. R., Bouvier, J., \& Martín, E. L. 2001, ApJ, 546, 1006

Barrado y Navascués, D., Stauffer, J. R., Briceño, C., et al. 2001, ApJS, 134, 103

Barrado y Navascués, D., Stauffer, J. R., et al. 2002, in preparation

Basri, G., \& Martín, E. L. 1999, 510, 266

Basri, G., Marcy, G. W., \& Graham, J. R. 1996, ApJ, 458, 600

Basri, G. 2000, ARA\&A, 38, 485

Béjar, V., Martín, E. L., Zapatero Osorio, M. R., et al. 2001, ApJ, 556, 830

Bouvier, J., Stauffer, J.R., Martín, E. L., et al. 1998, A\&A, 336, 490

Briceño, C, Hartmann, L., Stauffer, J. R., \& Martín, E. L. 1998, AJ, 115, 2074

Burrow, et al. 1997, ApJ, 491, 856

Chabrier, G., Baraffe, I., Allard, F., \& Hauschildt, P. 2000, ApJ, 542, L119

D’Antona, F., \& Mazzitelli, I. 1994, ApJS, 90, 467

D’Antona, F., \& Mazzitelli, I. 1997, in Cool Stars in Clusters and Associations, ed. R. Pallavicini, \& G. Micela, Mem. Soc. Astron. Italiana, 68(4), 807

Festin, L. 1997, A\&A, 322, 455

Hodgkin, S. T., \& Jameson, R. F. 2000, in Stellar Clusters and Associations: Convection, Rotation, and Dynamos, ed. R. Pallavicini, G. Micela, \& S. Sciortino, ASP Conf. Ser., 198, 245

Jeffries, R. D., Thurston, M. R., \& Pye, J. P. 1997, MNRAS, 287, 350

Jeffries, R. D., James, D. J., \& Thurston, M. R. 1998, MNRAS, 300, 550
Jeffries, R. D., Thurston, M. R., \& Hambly, N. C. 2001, A\&A, 375, 863

Jameson, R. F. 2002, in Brown Dwarfs, IAU Symp. 211, ed. E. L. Martín, in press

Koester, D., \& Reimers, D. 1996, A\&A, 313, 810

Kumar, S. S. 1963, ApJ, 137, 1121

Landolt, A. 1992, AJ, 104, 340

Leggett, S. 1992, ApJS, 82, 351

Lucas, P. W., \& Roche, P. F. 2000, MNRAS, 314, 858

Luhman, K. L. 1999, ApJ, 525, 466

Luhman, K. L. 2000, ApJ, 544, 1044

Martín, E. L., Brandner, W., Bouvier, J., et al. 2000, ApJ, 543, 299

Martín, E. L., Zapatero Osorio, M. R., Barrado y Navascués, D., Béjar, V., \& Rebolo, R. 2001, ApJ, 558, 117

Meynet, G., Mermilliod, J.-C., \& Maeder, A. 1993, A\&AS, 98, 477

Monet, D., et al. 1992, AJ, 103, 638

Moraux, E., Bouvier, J., \& Stauffer, J. 2001, A\&A, 367, 211

Najita, J. R., Tiede, G. P., \& Carr, J. S. 2000, ApJ, 541, 977

Neuhäuser, R., \& Comeron, F. 1999, A\&A, 350, 612

Pinfield, D. J., Hodgkin, S. T., Jameson, R. F., et al. 2000, MNRAS, 313,347

Pinsonneault, M. H., Stauffer, J., Soderblom, D. R., King, J. R., \& Hanson, R. B. 1998, ApJ, 504, 170

Prosser, C. F. 1992, AJ, 103, 488

Prosser, C. F. 1994, AJ, 107, 1422

Prosser, C. F., Randich, S., Stauffer, J. R., Schmitt, J. H. M. M., \& Simon, T. 1996, AJ, 112, 1570

Prosser, C. F. 1998, Astron. Nachr., 319, 215

Rebolo, R., Martín, E. L., \& Magazzù, A. 1992, ApJ, 389, L83

Rebolo, R., Martín, E. L., Basri, G., Marcy, G. W., \& Zapatero-Osorio, M. R. 1996, ApJ, 469, 706

Rebolo, R., Zapatero Osorio, M. R., \& Martín, E. L. 1995, Nature, 377,129

Rieke, G. H., \& Lebofsky, M. J. 1985, ApJ, 288, 618

Robichon, N., Arenou, F., Mermilliod, J.-C., \& Turon, C. 1999, A\&A, 345,471

Santiago, B. X., Gilmore, G., \& Elson, R. A. W. 1996, MNRAS, 281, 871

Siess, L., Dufour, E., \& Forestini, M. 2000, A\&A, 358, 593

Skrutskie M. F., Schneider S. E., Stiening R., et al. 1997, in The Impact of Large Scale Near-IR Sky Surveys, ed. F. Garzon et al. (Dordrecht: Kluwer Academic Publishing Company), 25

Stauffer, J. R., Hartmann, L. W., Burnham, J. N., \& Jones, B. F. 1985, ApJ, 289, 247

Stauffer, J. R., Hartmann, L. W., \& Jones, B. F. 1989, ApJ, 346, 160

Stauffer, J. R., Schultz, G., \& Kirkpatrick, J. D. 1998, ApJ, 499, L199

Stauffer, J. R., Barrado y Navascués, D., Bouvier, J., et al. 1999, ApJ, 527,219

Taylor, B. J. 1986, ApJS, 60, 577

Ventura, P., Zeppieri, A., Mazzitelli, I., \& D’Antona, F. 1998, A\&A, 334, 953

Wainscoat, R. J., Cohen, M., Volk, K., Walker, H. J., \& Schwartz, D. E. 1992, ApJS, 83, 111

Zapatero Osorio, M. R., Rebolo, R., Martín, E. L., \& García López, R. 1996, A\&A, 305, 519

Zapatero Osorio, M. R. 1997, Ph.D. Universidad de La Laguna, Spain

Zapatero Osorio, M. R., Rebolo, R., \& Martín, E. L., et al. 1999, A\&AS, 134, 537

Zapatero Osorio, M. R., Béjar, V. J. S., Martín, E. L., et al. 2000, Science, 290, 103

Zapatero Osorio, M. R., et al. 2002, private communication 